\title{
Antimicrobial Resistance Pattern of Pseudomonas aeruginosa of Various Clinical Samples in Government Medical College, Aurangabad, India
}

\author{
Shaikh Ambreen Fatema Abdul Hafiz, Anil A. Gaikwad*, J.A. Iravane, \\ Harbade (Duthade) and A.S. Damle \\ Department of Microbiology, Government Medical College, Aurangabad, India \\ *Corresponding author
}

\begin{tabular}{|l|}
\hline Key w or d s \\
$\begin{array}{l}\text { Pseudomonas } \\
\text { aeruginosa }(P . \\
\text { aeruginosa), ESBL, } \\
\text { AmpC, MBL }\end{array}$ \\
\hline Article Info \\
\hline $\begin{array}{l}\text { Accepted: } \\
\text { 17 January } 2018 \\
\text { Available Online: } \\
\text { 10 February } 2018\end{array}$ \\
\hline
\end{tabular}

A B S T R A C T

In this era spread of multidrug resistance is a global problem. $P$. aeruginosa is one of the most common nosocomial pathogen causing wide range of opportunistic infections. Rapid emergence of varying degrees of innate $\&$ acquired resistance mechanisms has put forward a major challenge in their detection as well as treatment options. We did a prospective study, from January 2016 to December 2016, in Department of Microbiology at Government Medical College, Aurangabad. Out of 3,340 culture positive samples, 676 (20.23\%) were identified as $P$. aeruginosa by standard bacteriological identification methods, from various specimens. Antimicrobial sensitivity testing was done by Modified Kirby-Bauer disc diffusion method as per CLSI guidelines 2016. Results showed maximum antibiotic resistance to Nalidixic acid (100\%), Ampicillin (83.3), Ceftriaxone (72.8\%), Nitrofurantoin (72\%), Cefepime (62.9\%), Norfloxacin (65.2\%), Cefotaxime (57.6\%), Ceftazidime (56.1\%) against Colistin (94.9\%), Amikacin (93.9\%), Piperacillin/Tazobactam (94.3\%), Imipenem (82.2\%), Cefoxitin (67.6\%) Ceftazidime/Clavulanic acid (57.9\%) which were identified as the most effective antibiotics. Prevalence of ESBL, AmpC and MBL producers was found to be $21.74 \%$, $10.62 \%$ and $9.31 \%$ respectively. Conclusion: Emerging multi drug resistant $P$. aeruginosa needs inevitable screening and confirmation for $\beta$ - lactamases production so that proper treatment can be initiated with appropriate antibiotics for commendable outcome, further their spread can be prevented by applying infection control policy and judicious use of antibiotics in the hospital.

\section{Introduction}

$P$. aeruginosa is an opportunistic pathogen that causes severe invasive diseases in critically ill patients or immunocompromised patients. The frequency of infections caused by them is increasing and multidrug-resistant (MDR) strains, resistant to almost all available antibiotics are emerging in hospitalized patients (Ozer et al., 2009). Restricted permeability and efflux are common components of the resistance phenotype for $\beta$ lactams, aminoglycosides and quinolones which are essentially fundamental properties of the organism. $P$. aeruginosa innate antibiotic resistance results from the restricted 
permeability of the cell wall. Resistance to $\beta$ lactams involves several mechanisms, the most prevalent mechanism is the production of $\beta$-lactamases, followed by permeability alterations, extrusion by efflux pumps, and to a lesser extent PBP alterations. Acquired resistance mechanism includes plasmid mediated AmpC $\beta$-lactamase, extended spectrum $\beta$-lactamase and metallo $\beta$ lactamase (MBL) enzymes (Manchanda and Singh, 2008).

A multidrug-resistant phenotype produces serious infections and often leaves us with only few reliable therapeutic options which have more side effects. As a result these types of infections are always associated with increased morbidity and mortality.

Emergence of multiple antibiotic resistance is a growing clinical concern, hence there is a need to conduct studies to profile resistance patterns in various hospitals so as to generate data that would guide clinicians to use antibiotics judiciously for treatment.

The main aim and objectives of this study to isolate and identify $P$. aeruginosa from various clinical samples. And to study the antibiotic profile of $P$. aeruginosa. Also to detect various type of $\beta$-lactamases producing $P$. aeruginosa.

\section{Materials and Methods}

We did a one year prospective study from January 2016 to December 2016 in Department of Microbiology at Government Medical College, Aurangabad. Various samples like blood, wound swabs/ pus, urine, sputum and tracheal aspirate were taken from hospitalized patients of different wards were evaluated and included in our study. In 2016, total number of culture positive isolates were 3,340; among that $676(20.23 \%)$ were $P$. aeruginosa. Identification of bacterial isolates was done by conventional bacteriological methods such as colony morphology, gram stain, catalase test, oxidase test, motility test, IMVIC tests and production of various pigments (pyocyanin, pyoverdin, pyomelanin and pyorubrum). Antibiotic susceptibility test was done by Modified Kirby-Bauer disk diffusion method on Muller Hinton Agar as per CLSI guidelines 2016 for various antibiotics, namely:

Colistin (10 $\mu \mathrm{gm})$, Ciprofloxacin (5 $\mu \mathrm{gm})$, Norfloxacin (10 $\mu \mathrm{gm})$, Nalidixic Acid (30 $\mu \mathrm{gm})$, Nitrofurantoin $(300 \mu \mathrm{gm})$, Ceftazidime/ Clavulanic acid (30/10 $\mu \mathrm{gm})$, Ceftazidime $(30 \mu \mathrm{gm})$, Cefepime $(30 \mu \mathrm{gm})$, Ceftriaxone $(30 \mu \mathrm{gm})$, Cefoxitin $(30 \mu \mathrm{g})$, Ampicillin (10 $\mu \mathrm{gm})$, Imipenem $(10 \mu \mathrm{gm})$, Amikacin $(30 \mu \mathrm{gm})$, Piperacillin+tazobactum (100/10 $\mu \mathrm{gm})$ (Clinical and Laboratory Standards Institute, 2016).

ESBL was detected by using Double disc synergy test in which difference of $\geq 5 \mathrm{~mm}$ between the zone diameters of ceftazidime and ceftazidime/clavulanate disks is seen, as per the CLSI 2016 guidelines. (Clinical and Laboratory Standards Institute, 2016) (As shown in Figure 1a).

AmpC $\beta$-lactamase production was tested by Disk Antagonism Test. Ceftriaxone $(30 \mu \mathrm{g})$ and cefoxitin $(30 \mu \mathrm{g})$ disks were placed 20 $\mathrm{mm}$ apart from centre to centre. Isolates showing blunting of the Ceftriaxone zone of inhibition adjacent to the cefoxitin disk were labelled AmpC $\beta$-lactamase producer (Upadhyay et al., 2010) (Fig. 1b).

MBL production was detected by Disk Potentiation Test in which Imipenem $(10 \mu \mathrm{g})$ and Imipenem-EDTA $(10 / 50 \mu \mathrm{g})$ were placed on the plate and the inhibition zones were compared after 16 to18 hours of incubation at $35{ }^{\circ} \mathrm{C}$. If the increase in inhibition zone with Imipenem - EDTA disk 
was $\geq 7 \mathrm{~mm}$ as compared to the Imipenem disk alone was considered to be the MBL producer (Yong et al., 2002) (Fig. 1c).

\section{Results and Discussion}

Out of $676 P$. aeruginosa in our study; maximum isolates $405(59.91 \%)$ were isolated from wound swab; 81 (11.98\%) from tracheal secretion; 67 (9.91\%) from urine; 61 (9.02\%) from sputum; 38 (5.62\%) from pus and 24 (3.55\%) from blood. Among infected patients, males infected were $58.43 \%$ and female $41.57 \%$. $P$. aeruginosa infection was seen in all age groups but rate of isolation was seen more in age group of $41-50$ years.

In our study $P$. aeruginosa showed maximum antibiotic resistance to Nalidixic acid (100\%), Ampicillin (83.3), Ceftriaxone (72.8\%), Nitrofurantoin (72\%), Cefepime (62.9\%), Norfloxacin (65.2\%), Cefotaxime (57.6\%), Ceftazidime (56.1\%) against Colistin (94.9\%), Amikacin (93.9\%), Piperacillin/Tazobactam (94.3\%), Imipenem (82.2\%), Cefoxitin (67.6\%) and Ceftazidime/Clavulanic acid $(57.9 \%)$ which were identified as the most effective antibiotics (Table 1).

In our study we got $229 \quad(33.87 \%) \quad P$. aeruginosa sensitive to all applied drugs, 72 (10.65\%) monoresistant or biresistant $P$. aeruginosa, $198 \quad(29.28 \%)$ MDR $P$. aeruginosa and 38 (5.6\%) were resistant to all drugs.

As shown in the (Table 2), out of 676 isolates of $P$. aeruginosa detected from various clinical samples $21.74 \%$ were ESBL producers, $10.65 \%$ were AmpC producer and 9.31\% were MBL producer. Among all the clinical specimens wound swab showed highest percentage of ESBL, AmpC and MBL producer. Co-existance of resistance mechanisms of $P$. aeruginosa were also studied. Only $0.44 \%$ isolates showed coexistence of ESBL, Amp $\mathrm{C}$ and MBL (Table 3).

$P$. aeruginosa has emerged as an important nosocomial pathogen as it is inherently resistance to many antimicrobial agents and antiseptics mainly due to synergy between multidrug efflux system and low outer membrane permeability (Manchanda and Singh, 2008). From the present study we observed that $P$. aeruginosa isolated from the burn patients was the most important cause of hospital acquired infection. $P$. aeruginosa isolates which were isolated in our institute showed remarkable resistance against penicillin and fourth generation cephalosporin. But the extended spectrum penicillin and the $3^{\text {rd }}$ generation cephalosporin in combination with Tazobactum and clavulinic acid showed a significant decrease in resistance pattern as it expands the spectrum of activity, the dose as well as incidence of toxicity reduces subsequently. The increased prevalence of resistance to cefepime reflects the increased use, rather we can say misuse of this drug in recent years.

Carbapenems are $\beta$ lactam antibiotics, presently considered as the most potent agents for the treatment of multidrug resistance pseudomonas infections as these agents are stable and have high rate of permeation through bacterial outer membranes. Carbapenem hydrolyzing MBL's have been reported in several countries and have emerged as the most important mechanisms of carbapenem resistance. Production of MBL by $P$. aeruginosa has tremendous consequences, since these organisms also carry other multi drug resistance genes and the only treatment option remains with us is potentially toxic colistin. 
Table.1 Antibiogram of $P$. aeruginosa

\begin{tabular}{|l|}
\multicolumn{1}{|c|}{ Antibioticname } \\
\hline Colistin \\
\hline Amikacin \\
\hline Piperacillin/Tazobactam \\
\hline Imipenum \\
\hline Cefoxitin \\
\hline Ceftazidime/Clavulanic acid \\
\hline Ceftazidime \\
\hline Cefotaxime \\
\hline Ciprofloxacin \\
\hline Norfloxacin \\
\hline Cefepime \\
\hline Nitrofurantoin \\
\hline Ceftriaxone \\
\hline Ampicillin \\
\hline Nalidixic acid \\
\hline
\end{tabular}

\begin{tabular}{|r|r|}
\hline \multicolumn{1}{|l|}{ \% } & \multicolumn{1}{|c|}{ \% } \\
\hline 94.9 & 5.1 \\
\hline 93.9 & 6.1 \\
\hline 94.3 & 5.7 \\
\hline 82.2 & 17.8 \\
\hline 67.9 & 32.1 \\
\hline 57.9 & 42.1 \\
\hline 43.9 & 56.1 \\
\hline 42.4 & 57.6 \\
\hline 37.9 & 62.1 \\
\hline 34.8 & 65.2 \\
\hline 37.1 & 62.9 \\
\hline 28 & 72 \\
\hline 27.2 & 72.8 \\
\hline 16.7 & 83.3 \\
\hline 0 & 100 \\
\hline
\end{tabular}

Table.2 Number of isolation among various Beta-Lactamase producer

\begin{tabular}{|l|}
\hline Total no. \\
\hline 676 \\
\hline \\
\hline 1. \\
\hline 2. \\
\hline 3. \\
\hline 4. \\
\hline
\end{tabular}

\begin{tabular}{|l|l|}
\hline ESBL & AmpC \\
\hline $147(21.74 \%)$ & $72(10.65 \%)$ \\
\hline
\end{tabular}

MBL

of isolates

$147(21.74 \%)$

Table.3 Coexistence of ESBL, Amp C and MBL

\section{Multiple resistance}

$\mathrm{ESBL}+\mathrm{MBL}$

$\mathrm{ESBL}+\mathrm{AmpC}$

$\mathrm{MBL}+\mathrm{AmpC}$

$\mathrm{ESBL}+\mathrm{AmpC}+\mathrm{MBL}$

\section{Number (\%) \\ $18(2.66 \%)$ \\ $26(3.8 \%)$ \\ $13(1.92 \%)$ \\ $3(0.44 \%)$}

Fig.1 $P$. aeruginosa showing production of various type of $\beta$-lactamases

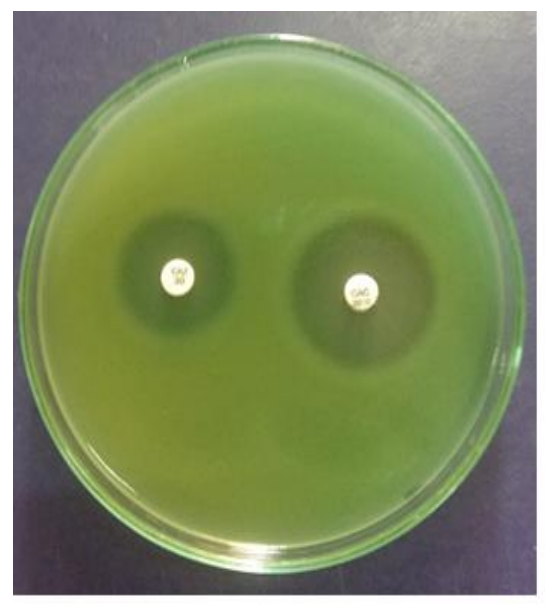

Fig 1(a): Positive Double disc synergy test

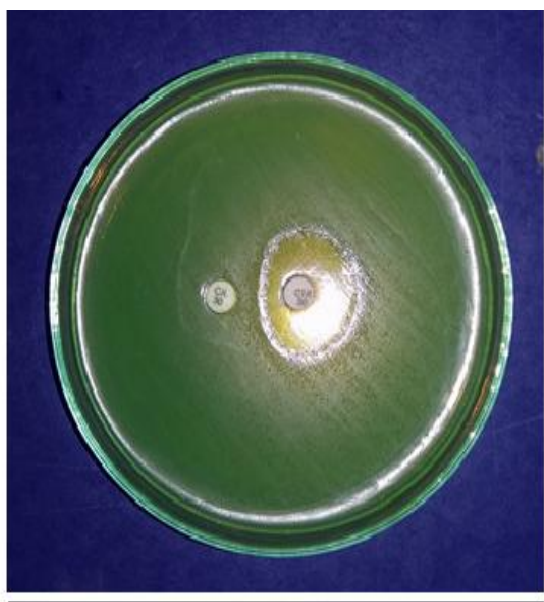

Fig 1(b): Positive Disc Antagonism Test

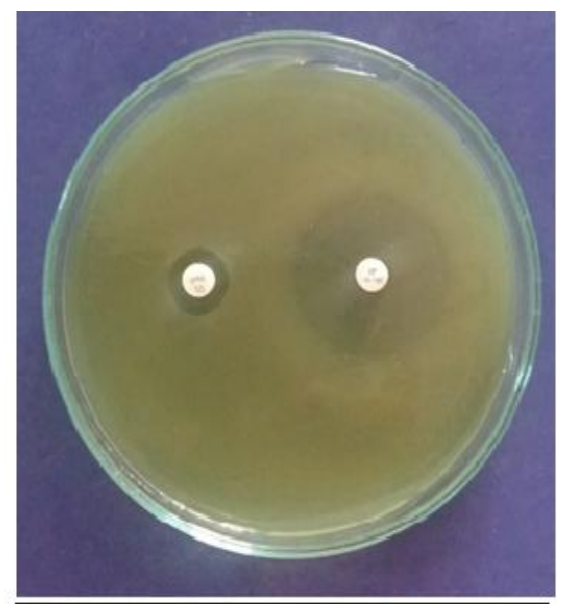

Fig 1 (c): Positive Disc Potentiation Test 
In our study Piperacillin Tazobactum, Amikacin and colistin were highly effective against MBL producers. Better anti pseudomonal activity with colistin, piperacillintazobactum, amikacin and imipenem goes in accordance with various studies published (Chaudhari et al., 2013; Amutha et al., 2009; Joseph et al., 2013; Senthamarai et al., 2014).

In the present study $21.74 \%$ isolates were ESBL producers and $10.65 \%$ Amp C $\beta$ lactamases producers similar to study done by Umadevi et al., (19.4\%) and (16.4\%) but MBL in our study was found to be $9.31 \%$ which is very low as compared to Umadevi et al., which reported $65.7 \%$ (Umadevi et al., 2011). A study conducted at NIMS Hospital, Rajasthan by (Gagan Priya Pandey et al., 2017) showed lower incidence of $\beta$ lactamases production as compared to our study. They studied 50 isolates in which $8 \%$ showed ESBL production, $8 \%$ showed AmpC production and $6 \%$ showed MBL production (Gagan Priya Pandey et al., 2017).

We have also studied the coexistence between ESBL - Amp C, Amp C - MBL, ESBLMBL and ESBL- Amp C - MBL in isolates of $P$. aeruginosa. In our study coexistence between $\mathrm{MBL}+\mathrm{Amp} \mathrm{C}$ was found to be $1.92 \%$ which is similar to study conducted by (Gagan Priya Pandey et al., 2017) showing $2 \%$. Whereas coexistence between ESBL Amp C, ESBL- MBL and ESBL- Amp C MBL in isolates of $P$. aeruginosa were not reported in (Gagan Priya Pandey et al., 2017) and we reported $2.6 \%, 3.8 \%$ and $0.44 \%$ respectively.

In case of pan drug resistance, colistin along with $\beta$ lactams was advised in our institute to patients as per National treatment guidelines (National treatment Guidelines, 2016) which successfully treated the patients. In using combination therapy probability increases that the infecting pathogen will be susceptible to at least one of the antibiotics; plus there is synergistic effect of the combination which prevents emergence of drug resistance.

Widespread use of antibiotics has altered etiological pattern of infections and their antibiotic susceptibility. Hence continuous monitoring of susceptibility pattern needs to be carried out in individual setting so as to detect the true burden of antibiotic resistance among organisms and prevent their further emergence by judicious use of drugs (Kaur et al., 2008).

This study was done for the first time in our institute, as multi drug resistance producing organisms are emerging, it becomes our responsibility as microbiologist to screen and confirm for $\beta$ - lactamases production of every sample so that proper treatment can be initiated with appropriate antibiotics for favourable outcome. It should be made mandatory for all clinician to send all the samples for microbiological analysis and their antibiogram before starting antibiotics so that emergence of drug resistance can be minimised. Control measures which include the judicious use of antibiotics, antibiotic cycling, and the implementation of appropriate infection control measures and the formulation of an antibiotic policy must be done to prevent the spread of $P$. aeruginosa infection.

\section{References}

Amutha R, Padmakrishnan, Murugan T, Renugadevi MP. Studies on multidrug resistant Pseudomonas aeruginosa from pediatric population with special reference to extended spectrum beta lactamase. Indian Journal of Science and Technology 2009; 2(11):11-13

Chaudhari VL, Gunjal SS, Mehta M. Antibiotic resistance patterns of 
Pseudomonas aeruginosa in a tertiary care hospital in Central India. IntJMedSci Public Health 2013; 2:386389

Clinical and Laboratory Standards Institute. Performance standards for antimicrobial susceptibility testing. CLSI guideline M100S-26. Wayne, P.A: CLSI, 2016

Gagan Priya Pandey, Akansha Rana, Dr Kush Manna NKK. Detection of Various Beta Lactamases in Pseudomonas aeruginosa from. JMSCR. 2017; 5(1):15527-35.

Joseph NM, Devi S, Shashikala P, Kanungo R. Changing Trend in the Antibiotic Resistance Pattern of Pseudomonas aeruginosa Isolated from Wound Swabs of Out-Patients and in-Patients of a Tertiary Care Hospital. Journal of Clinical and Diagnostic Research. 2013 Oct; 7(10): 2170-2

Kaur J, Gulati VL, Aggarwal A, Gupta V. Bacteriological profile of osteomyelitis with special reference to Staphylococcus aureus. Indian Journal for the Practising Doctor. 2008; 4:6.

Manchanda V, and Singh NP 2008. Occurrence and detection of AmpC blactamases among Gram negative clinical isolates using a modified threedimensional test at Guru TeghBahadur Hospital, Delhi, India. J Antimicrob Chemother 51: 415-418.

National treatment Guidelines for Antimicribial Use in infectious diseases Version 1.0 (2016)
Ozer, B., M. Tatman-Otkun, D. Memis and M. Otkun, "Cha- racteristics of Pseudomonas aeruginosa Isolates from In-tensive Care Unit," Central European Journal of Medi-cine, Vol. 4, No. 2, 2009, pp. 156-163.

Senthamarai S, Suneel Kumar Reddy A, Sivasankari S, Anitha C, Somasunder V, Kumudhavathi MS, Amshavathani SK, Venugopal V. Resistance Pattern of Pseudomonas aeruginosa in a Tertiary Care Hospital of Kanchipuram, Tamilnadu, India. Journal of Clinical and Diagnostic Research. 2014; 8(5):30-2

Umadevi S, Joseph NM, Kumari K, Easow JM, Kumar S, Stephen S, Srirangaraj S and Raj S: Detection of extended spectrum beta lactamases, AmpC beta lactamases and metallobetalactamases in clinical isolates of ceftazidime resistant Pseudomonas aeruginosa. Braz J Microbiol. 42: 1284-1288. 2011. Upadhyay S, Sen MR, Bhattacharjee A. Presence of different beta-lactamase classes among clinical isolates of Pseudomonas aeruginosa expressing AmpC beta-lactamase enzyme.J. Infect DevCtries 2010; 4(4): 239-242

Yong D, Lee K, Yum JH, Shin HB, Rossolini GM, Chong Y 2002. Imipenem EDTA disk method for differentiation of metallo ßlactamase producing clinical isolates of Pseudomonas spp. and Acinetobacter spp. J ClinMicrobiol 40: 3798801

\section{How to cite this article:}

Shaikh Ambreen Fatema Abdul Hafiz, Anil A. Gaikwad, J.A. Iravane, Harbade (Duthade) and Damle, A.S. 2018. Antimicrobial Resistance Pattern of Pseudomonas aeruginosa of Various Clinical Samples in Government Medical College, Aurangabad, India. Int.J.Curr.Microbiol.App.Sci. 7(02): 1662-1667. doi: https://doi.org/10.20546/ijcmas.2018.702.201 